\title{
Flocart et Florimont, le miroir et le prince
}

Florimont, d'Aimon de Varennes, un trouvère lyonnais du XII ${ }^{e}$ siècle, semble avoir été écrit en 1188, mais sa datation demeure incertaine. Ce roman narre comment un jeune homme, profondément épris d'une fée, est amené à trahir malgré lui celle qu'il aime, perdant ainsi son amour et comment, après bien des souffrances, le jeune héros se console auprès d'une autre et devient roi. Tout au long des aventures de Florimont, son maitre Flocart l'accompagne, le guide et l'éduque par des discours fortement moralisateurs.

Nous nous proposons d'étudier comment ce personnage qui, selon Katalin Halász, " participe en quelque sorte d'un pouvoir supérieur, ou au moins de l'omniscience du narrateur » (1992, p. 12) construit sa relation avec son élève, dans un but d'édification individuelle et collective.

Nous verrons tout d'abord que Flocart assume une double fonction diégétique qui favorise le processus d'individuation de son élève. Nous montrerons ensuite que ses multiples interprétations des rêves lui confèrent le rôle de " maître de vérité » (Bonnet, 2013 , p. 8) qui sacralise le destin de Florimont. Nous observerons, enfin, que les discours parémiologiques de Flocart prennent des allures de miroir aux princes propre à révéler à son jeune élève les principales qualités d'un bon roi en plaçant la largesse au cœur des relations humaines et politiques.

- Nathalie Leclercq - docteur ès lettres, agrégé de lettres modernes, membre non permanent du CERAM de l'Université Sorbonne Nouvelle - Paris 3, enseignante au lycée Pierre Bourdan de Guéret : B.P. 19 Place Molière 23011 Guéret cedex. Adresse de correspondance : leclercqn@me.com ORCID iD : https://orcid.org/0000-0002-5690-5189 


\section{Une double fonction diégétique}

Les vers 1962 à 2666 narrent comment Florimont tua un monstre avec une tête de léopard et un corps de guivre, qui, envoyé par Garganeüs, dévastait les terres du duc. Une fois la bête abattue, le jeune héros aperçut un palefroi et une magnifique jeune fille, la fée de l'Île Celée, qui le remercia d'avoir occis l'assassin de son père, de sa sœur et de son frère. Elle lui proposa de devenir son époux, mais le jeune homme refusa car, malgré l'amour qu'il ressentait pour la fée, il se devait de retourner chez son père et sa mère afin de ne pas les laisser sans nouvelles de lui. La fée accepta son départ et lui assura qu'il pourrait la voir quand il le voudrait, à la seule condition qu'il vienne toujours seul, sinon il risquait de la perdre à jamais. Flocart, grâce à ses dons divinatoires, découvrit cette relation :

Floquars l'ait bien aperseü

Per nigromance [et] coneü

Que o li s'en veloit aler,

A la duchesce vet parler : [...] (v. 3683-3686)

Ce pouvoir supérieur et sa faculté d'anticipation lui conférèrent une omniscience qui le poussa à contrecarrer les projets amoureux de son jeune élève en révélant à la mère de Florimont toute la vérité :

«Dame, ce dist Floquars, per foi,

J'ai grant paor. » - « Maistre, de coi ?»

«Que mes sires ne soit perdus.

Femes ont atres deseüs.

Je croi bien que il ait trovee

La pucele d'Île Selee.

C’ele len puet mener o soi,

Sertes, nel vairés mais, se croi. » (v. 3687-3694)

À l'instar du narrateur, Flocart fait figure de fin rhétoriqueur et manie autant l'analepse ${ }^{1}$ que la prolepse $e^{2}$ pour faire craindre à la mère les conséquences futures d'un passé dommageable. Même si ses motivations demeurent d’ordre politique et social (loin de son royaume, Florimont ne serait ni en mesure d'aider son père à gouverner, ni capable d'assumer ses devoirs de chevalerie), Flocart semble ainsi

1. «En narratologie et en dramaturgie : technique qui consiste à rompre le fil chronologique du récit (ou du cours des actions au théâtre), par la version de détails antérieurs, mais aussi d'un épisode entier ou d'un texte » (Van Gorp et al., 2005, p. 33).

2. En narratologie, anticipation narrative qui vient rompre le parallélisme entre l'ordre du récit et celui des événements qui constituent l'histoire (Van Gorp et al., 2005, p. 389). 
l'incarnation de Fortune en forçant le destin de son jeune protégé. En outre, ce récit enchâssé relance l'action et fait tourner la roue de la Fortune que le narrateur évoque quelques vers auparavant :

Mai Fortune sa roe vire :

Quant sor la roe la veü,

Si l'en a mout tost abatu.

Maudite soit hui sa fiance

Et sa roe que si balance! (v. 3674-3678)

En effet, la reine suivit son fils à son insu et se montra à la pucelle qui disparut à jamais. Flocart revêt donc une double fonction diégétique. Premièrement, au niveau macrostructural du récit ${ }^{3}$, le maître fait figure d'auteur en donnant une orientation différente aux aventures. Sans son intervention, l'action aurait bifurqué dans une toute autre direction, Florimont n'aurait jamais trahi celle qu'il aime. De surcroît, Flocart agit également au niveau microstructural du récit en manipulant le développement émotionnel et psychologique de son jeune élève qui, fou de douleur, se métamorphose en "Pauvre Perdu ». Cette étape semble nécessaire au processus d'individuation de Florimont qui s'entend comme

l'élaboration des signes de l'identité personnelle (spécialement le nom, la signature et le portrait) [...] [et est] constituée d'un ensemble d’étapes dont la période médiévale constitue un moment bien particulier : l'individu sous ses différentes facettes (l'acteur social ; lêtre moral, indépendant, autonome ; le « je » des poètes soucieux d'introspection) s'exprime à la première personne sans pour autant se singulariser ni se démarquer du groupe qui le définit (famille, lignage, paroisse, seigneurie...). (Bedos-Rezak, 2005, p. 1)

Florimont définit lui-même sa nouvelle identité :

Quant on l'apele : « Florimont!»

A mout grant poengne lor respont.

«Signor, fet il, tort en avez

Quant vos Florimont m'apalez :

De Florimont nen i a mie ;

Il s'en ala avec s'amie

Quant em sopirant la baissa,

Mai por Florimont sai laissa

3. Modèle créé par Teun A. Van Dijk et Walter Kintsch qui offre la représentation schématique d'un texte dont le "résumé » qu'effectue notre cerveau pour le retenir en recourant à des mécanismes de suppression, de généralisation, d'intégration, favorise son appréhension dans sa totalité (Van Dijk et Kintsch, 1983, p.47 et ss.). 
Un chevelier povre perdu.

Ne l'avez vos donc coneü?» (v. 4013-4022)

La subjectivité du personnage qui se joue du sémantisme du participe passé per$d u$ pour souligner sa perte identitaire imprègne ce discours à la première personne. Si la folie d'amour paraît un motif traditionnel du récit courtois, ce traitement trouve son originalité dans la narration que propose le personnage de ses propres aventures et dans ses explications. Qu'il s'agisse d'une vérité ou d'un mensonge inconscient (Florimont connait-il encore son nom ?), le discours analeptique rend compte d'une fêlure dans la personnalité du jeune homme, d'une cassure, que seule Romadanaple s'avèrera en mesure de guérir. En provoquant la crise identitaire de son élève, Flocart lui a permis d'accéder à la vérité de l'amour et de surmonter ses ravages dévastateurs afin d'accomplir son destin royal.

\section{Un maître de vérité}

En outre, ses interprétations des rêves des autres personnages confèrent à Flocart le rôle de "maitre de vérité » (Bonnet, 2013, p. 8) et de double idéologique du narrateur qui détient un savoir, ou du moins des informations, dont la source reste le plus souvent indéterminée.

Dans leur introduction du Rêve médiéval, Alain Corbellari et Jean-Yves Tilliette précisent qu'il n'y a pas de " 'rêve médiéval', ou plutôt, [qu'] il n'y a pas de différence entre le rêve médiéval et le rêve tout court » (2007, p. 7-9). Il semble ainsi question " non du rêve, mais de rêveurs, réels ou fictifs, et des discours qui leur servent à traduire les images nocturnes venues les visiter » (p. 7-9).

Le père du héros, le roi Philippe, fait plusieurs rêves dont celui avant la naissance de Florimont qui sert de leitmotiv au roman et « d'outil solide pour construire un discours d'enseignement, [mais] un discours rusé qu'il faut analyser, déchiffrer pour ne pas être trompé » (Minet-Mahy, 2007, p. 205):

Avis li fut en (a)vision

Qu'il veoit un petit lyeon.

Li lyeonsiaus que il veoit

En son lit joste lui gissoit ;

Ne li faissoit mies paor,

Ainz li mostroit semblant d'amor. (v. 1715-1720)

Le songe conserve toute sa charge émotionnelle en maintenant la curiosité du lecteur/auditeur en éveil, les déterminants indéfinis et l'indétermination du cadre spatio-temporel intensifiant l'ignorance du rêveur et son incapacité à comprendre toute la portée de son rêve, qui se poursuit sur une centaine de vers : 
Donc s'en aloit vers ses boscaiges

Et trovoit ses bestes savaiges

Et si trovoit un grant lyeon

Que faissoit de lui compaignon. (v. 1727-1730)

Le rêve devient une « activité culturelle codifiée par une collectivité qui imprime ainsi sa marque sur les discours qu'elle produit » (Parrot, 1995, p. 89). Même si le lecteur/auditeur ne pénètre pas encore toute la signification de ce récit, il en comprend la portée allégorique.

Philippe confie la mise en écrit de son rêve et son interprétation à Flocart, personnage clef présenté comme le millor maistre (v. 1862) de la cour :

Mout avoit apris en s'emfance

Astronomie et nigromance

Et savoit de dialetique,

De gramaire et de musique

Et de retorique savoit,

De fisique asi s'entendoit ;

Tot savoit quanqu'il a mestier

Ou a clerc ou a chevelier. (v. 1865-1872)

Il devient tout d'abord le double du narrateur par son savoir et sa science grâce auxquels il devine la réelle portée du rêve, mais aussi le double de l'auteur lui-même quand il met le rêve à l'écrit au fur et à mesure que Philippe le raconte :

La vission li conte et dist,

Et [c]i1 l'ait tot mis en escrit. (v. 1877-1878)

Enfin, en différant la révélation de la signification du rêve, Flocart en conserve toute la puissance énigmatique et la force évocatrice. Il convoque également les facultés inférentielles ${ }^{4} \mathrm{du}$ lecteur/auditeur et sa capacité d'interprétation cognitive :

Li maistres dire ne veloit

De l'emfant seu qu'il en savoit,

Mai tant li dist : « Enjandrei ais

Un enfant cui mout amerais.

4. « Le sujet humain possède la capacité d'élaborer, à partir d'informations primitives sur létat de son environnement, d'autres informations sur ce même environnement par des activités totalement intériorisées. Ces activités sont dites inférentielles, et les informations dérivées sont dites inférées. L’activité et le résultat sont communément appelés inférence, les énoncés constituant les informations initiales sont appelés prémisses. On distingue, au sein des activités inférentielles, le raisonnement déductif et le raisonnement inductif» (Borillo, 2002, p. 100). 
Garde le bien quant il iert neis ;

Que mal et bien avrait assez. » (v. 1879-1884)

La première relecture du rêve intervient après l'adoubement de Florimont en Bulgarie. Flocart devient ici un substitut du narrateur à part entière en décryptant le sens du récit. L'exégèse lui assigne une supériorité sur les autres personnages, à l'instar du narrateur, ses capacités de mise à distance des aventures et de leur interprétation lui conférant une fonction herméneutique. Ainsi, Flocart explicite toute la teneur symbolique du bestiaire mis en oeuvre ; le lionceau représente le jeune Florimont dont il annonce la dimension héroïque et royale :

Puels ait garde en son escrit :

Menbra li de la vission

Del lyeoncel et del lyeon

Que li dus li avoit conté.

Or sot il bien de verité :

Se fut li lyeonsiaus dorez

Quant Florimons vit adoubé. (v. 2946-2952)

Le lion s'avère " la 'star' du bestiaire médiéval » (Pastoureau, 1984, p. 133-142), un symbole positif, comme l'atteste par exemple le surnom de Richard " cour de Lion ». Selon Michel Pastoureau, le lion signifie le courage et la vaillance ; en témoigne le roman de Chrétien de Troyes, Yvain ou le chevalier au Lion : «Tous les bestiaires soulignent le courage du lion, sa générosité, son sens de la justice, toutes vertus qui sont le propre des rois » (2011, p. 62). L'animal met sa force au service du bien commun, son rugissement exprime la parole de Dieu (Pastoureau, 2011, p. 62). L'intégralité du rêve de Philippe revêt donc une portée proleptique, même s'il reste longtemps plus ou moins énigmatique.

La seconde interprétation du rêve par Flocart se situe lors du retour à Duras, après la victoire du jeune chevalier contre Garganeüs. La thématique de la lecture paraitt fondamentale dans le mécanisme de recognition ; Flocart semble bien le double du narrateur car il devient un intermédiaire entre l'histoire (les aventures de Florimont), sa narration et son interprétation :

Quant Garganeüs fut vancus

Et ses estaiges comfondus,

Floquars son escrit esgarda :

De la vision li menbra

Que li dit li dus del singler.

Dons sot il bien sens deviner

Que li lyeonsiaus avoit mort

Le singler que lor faissoit tort. (v. 3657-3664) 
Il demeure significatif que pour les Germains, affronter en combat singulier un ours ou un sanglier constitue pour tout jeune homme un rituel indispensable pour devenir un guerrier libre et adulte. Cependant, le sanglier incarne aussi tous les ennemis du Christ, symbolise l'antithèse du cerf : « aux dix 'propriétés' christologiques de celui-ci correspondent dix 'propriétés' diaboliques de celui-là » (Pastoureau, 2004, p. 75-78). L'épisode participe ainsi de l'héroïsation de Florimont et paraît d'autant mieux mis en valeur que c'est à Flocart - substitut diégétique du père - qu'en revient l'explication.

La troisième interprétation advient lorsque le Pauvre Perdu décide de suivre Rysus :

Si esgarda en son escrit :

Seu que li dus li avoit dit

Trovait del chetif lyeoncel

Qui n'avait que los et la pel

Et a grant veltre sen aloit

Et puels del levrier s'acoentoit.

Or sot Floquars de verité

Que li lyeonsiaus ot trové

Et le ve[1]tre et les levriers. (v. 4709-4717)

L'interprétation s'enclenche comme toujours par la mise en scène de relecture (esgarda en son escrit) et d'exégèse (mise en valeur par la répétition du verbe "savoir »). Elle suit toujours de près un événement majeur et conforte le lecteur/auditeur dans l'idée selon laquelle le rêve renferme bien une portée proleptique. Flocart comprend de suite l'importance de l'événement et met tout en œuvre pour aider son jeune maître à recevoir comme il se doit le nouveau venu.

Lors de la dernière aventure pendant laquelle Florimont délivre son père, le travail d'interprétation passe par le jeu alternatif des moments de tension (générés par la narration des rêves et tout le questionnement qu'ils suscitent) et ceux de détente (lorsque Flocart les explique et apporte des réponses aux interrogations) :

Floquars en son escrit esgarde,

De la vission se prist garde

Et si a trové del lyeon

Qui le duc getoit de prison

Et puels serchoit les fores tant

Que il conqueroit l'olifant.

Floquars voit l'olifant maté

Et le duc de prison geté :

Bien ait la vision trovee

Que li dus li avoit contee. (v. 13473-13482) 
Le verbe de vision esgarde et le verbe introspectif prist garde mettent en valeur les différents mouvements en marquant le désir d'interprétation, tandis que trove et voit (repris en écho par l'expression synthétique la vision trovee) rendent compte de l'analyse du rêve, son explication. Ce va-et-vient entre rêve, souhait de l'interprétation et interprétation produit cette "déclivité " ou ce " relief » qui confèrent au texte sa "pertinence " (Bronkart, 1985, p. 51). Les interprétations de Flocart servent de leitmotiv et confortent la portée allégorique du rêve pour peu que le lecteur/auditeur prenne « conscience du code» (Minet-Mahy, 2007, p. 212). Le songe apparaît alors comme métaphore du texte, et Flocart, double du narrateur, concrétise les rêves les plus fous en recourant au bestiaire pour allégoriser le destin de Florimont et lui conférer une dimension épique (il sort vainqueur des épreuves), politique (il devient le roi annoncé par la figure du lion), voire messianique (il devient le Sauveur de son royaume). Les exégèses du maître permettent à l'élève d'acquérir ainsi plus de profondeur, l'onirisme allant de pair avec une volonté de sacralisation du héros dont l'aura s'avère presque divine (Baroni, 2007, p. 49). Flocart fait ainsi figure magistrale de "passeur d'une vérité transcendante " (Bonnet, 2013, p. 8), ses interprétations confirmant "notre sentiment que [ce] personnage est un élu dont un pouvoir surnaturel suit la destinée avec une attention particulière » (Halász, 1992, p. 55).

\section{Un miroir pour un prince}

Flocart ponctue enfin ses conseils de discours parémiologiques qui prennent des allures de miroir aux princes propres à révéler à son jeune élève ses conceptions du bon roi et à rappeler ses principales qualités en plaçant la largesse au cœur des relations humaines et politiques.

Les miroirs aux princes ${ }^{5}$ existaient déjà durant l'Antiquité et se développèrent véritablement au VIII ${ }^{e}$ siècle ${ }^{6}$. Ils sont apparus au Moyen Âge sous la forme de traités d'éthique gouvernementale. Même si Florimont possède très peu de points com-

5. «Un 'miroir aux princes' se définit comme un traité écrit pour un prince - et en général dédié à lui - qui garde pour objet principal de décrire le prince idéal, son comportement, son rôle et sa situation au monde » (Már Jónsson, 2006, p. 153-166).

6. En Europe, le premier véritable miroir aux princes de lépoque carolingienne fut la Via regia écrite par Smaragde de Saint-Mihiel ((1853). Patrologie Latine (ou plus exactement Patrologiae Cursus Completus, Series Latina), Paris : Jacques-Paul Migne) aux alentours de l'année 813, et dont le destinataire semble être Louis le Pieux, alors qu'il n'était pas encore empereur. Parmi d'autres écrits de ce type, il convient aussi de citer le De regis persona et regio ministerio d'Hincmar de Reims rédigé en 873 ((1885). Patrologie Latine, 124, Paris : F. Vieweg), et le De institutione regia que Jonas d'Orléans écrivit vraisemblablement en 831 pour Pépin d'Aquitaine, un des fils de Louis le Pieux (Dubreucq, A. (éd.). (1995). Paris : Cerf, 407). 
muns avec un traité, Flocart se fait l'énonciateur de véritables discours politiques ${ }^{7}$ qui fondent une relation maître/élève essentiellement verticale reposant sur l'écoute, la confiance et une interaction féconde afin de former le jeune homme et de lui inculquer les principales qualités d'un bon roi.

Flocart consacre ainsi plus de cent vers ${ }^{8}$ à l'énumération des sept largesses, ponctuant son long discours de sentences et de proverbes qui personnifient et présentent cette qualité comme le «bon usage de [la] richesse prodigieuse » (Boutet, 1992, p. 262) du monarque. Le discours du personnage redouble celui du narrateur en sollicitant le fort pouvoir idéologique des sentences pour associer l'altruisme et la charité :

Qui tel largesce welt avoir

Son signor met en grant pooir

Tel largesce met son signor

De povrete en grant honor. (v. 4355-4358)

L'impersonnalisation modèle un personnage compétent et averti qui contrôle parfaitement les tenants et les aboutissants de la narration. Elle accentue également l'apparente objectivité du propos, d'autant que l'usage gnomique et parémiologique (Schulze-Busacker, 1997, p. 565-576) - dont Marie-Louise Ollier cerne la spécificité comme argument d'autorité importé(2000, p. 125-155) - tend à renforcer la maitrise et le savoir du maitre. Flocart se mue ainsi en triple figure de l'auctoritas, au niveau intradiégétique et extradiégétique dans « une tentative empirique de mettre le monde en ordre» (Meschonnic, 1976, p. 421).

Tout d'abord, au niveau intradiégétique, Flocart fait figure de maître omniscient qui éduque Florimont afin de l'aider à devenir un bon roi. Le fort pouvoir didactique du personnage trouve toute son efficacité dans la suite du récit puisque quelques aventures plus loin :

Li rois Florimons fut mout saiges,

Et mout l'amoit toz li barnaiges.

Or l'ot Fortune bien assis,

Et plus halt de sa roe mis.

Li petit et li grant l'amoient

Por la bonté qu'an lui veoient.

Per sa bonté, per sa largesce

Fut il montez en grant hatesce;

7. Voir notamment lorsque Flocart, à maintes reprises dans le récit, conseille Florimont avant qu'il ne devienne roi, et même après (ainsi au début du récit, lorsque Flocart envoie Florimont chez son oncle le roi d'Esclabonie, il lui donne de nombreux conseils : ne rien accepter, être généreux, se taire auprès des femmes, et il se livre à un long discours sur l'amour. Il lui recommande également de se garder du mauvais orgueil et de demeurer humble, v. 2723-2897).

8. Florimont, v. 4202-4358. 
De tote la gent fut ameis,

Et sa feme l'amoit asseis. (v. 11411-11420)

En outre, Flocart fait figure de double du narrateur en reprenant en écho les propos et les digressions de ce dernier qui placent la largesse au cœur des relations humaines et politiques. En effet, le narrateur loue également cette haute qualité :

Car largesce est meire d'amour

Et de proësce et de valour. (v. 95-96)

Le groupe ternaire complément du nom, d'amour/Et de proësce et de valour, assimile la valeur courtoise aux vertus chevaleresques, unissant les deux idéologies pour cerner l'image d'un roi modèle, détenteur de tous les attributs définis dans la tradition de l'augustinisme politique (Boutet, 1992, p. 167-169) ${ }^{9}$.

Enfin, au niveau extradiégétique, Flocart fait figure de double de l'auteur car celui-ci semble se servir de ces interventions pour rappeler aux seigneurs dont il dépend l'importance de la prodigalité et la façon dont les relations avec leur vassal doivent se fonder sur des rapports de " service mutuel, d'aide et d'amitié réciproques " (Gourevitch, 1983, p. 256). L'auteur conçoit donc son personnage tel un archétype afin de livrer un message aux seigneurs auxquels il s'adresse pour mieux les amandeir ${ }^{10}$, dans une hiérarchisation des vertus auxquelles ils doivent se conformer.

\section{Conclusion}

La relation maître/élève qu'entretiennent Flocart et Florimont se fonde sur un rapport d'autorité qui s'explique par l'omniscience et l'éloquence d'un personnage désireux de parfaire l'éducation tant individuelle que politique de son jeune disciple.

Flocart assume une double fonction diégétique en manipulant le destin de son élève pour favoriser le processus de son individuation. Il fait figure de maittre de vérité et de double de l'auteur lorsqu'il se mue en exégète des rêves des autres personnages dans une entreprise d'héroïsation, voire de mythification, du héros. Ses nombreux discours parémiologiques prennent enfin des allures de miroir aux princes dans un double projet d'édification intra et extradiégétique, l'espace romanesque se muant en miroir nitescent du monde réel de l'auteur.

9. Nous définissons l'« augustinisme politique » comme «la tendance à laisser l'Église absorber l'État, à ne considérer les affaires séculières qu’à travers leur subordination aux affaires de l'Église » (Arquillière, 1955, p. 4).

10. Voir le prologue de Florimont (Aimon Von Varennes, 1933, v. 42). 


\section{RÉFÉRENCES}

Aimon Von Varennes (1933). Florimont, ein altfranzösischer Abenteuerroman zum ersten Mal mit Einleitung, Anmerkungen, Namenverzeichnis und Glossar unter Benützung der von A. Risop gesammelten handschriftlichen Materialien herausgegeben von A. Hilka. Halle : Niemeyer.

Arquillière, H.-X. (1955). L’Augustinisme politique. Paris : Vrin.

Baroni, R. (2007). La Tension narrative. Suspense, curiosité et surprise. Paris : Seuil.

Bedos-Rezak, M., Iogna-Prat, D. (dir.). (2005). L'Individu au Moyen Âge. Individuation et individualisation avant la modernité. Paris : Aubier.

Bonnet, C., Marot, P., Charalampos, O., Noacco, C. (dir.). (2013). Figures du maître : De l'autorité à l'autonomie. Rennes : Presses Universitaires de Rennes.

Borillo, M., Goulette, J.-P. (dir.). (2002). Cognition et Création. Explorations cognitives des processus de conception. Bruxelles : Mardaga.

Boutet, D. (1992). Charlemagne et Arthur ou le roi imaginaire. Paris : Champion.

Bronkart, J.-P. (1985). Le Fonctionnement des discours. Neuchâtel : Delachaux et Niestlé.

Corbellari, A., Tilliette, J. Y. (éd.). (2007). Le Rêve médiéval. Genève : Droz.

Gilles de Rome. (1857). De Regimine principum doctrina (Traité sur le gouvernement des princes, vers 1280). Paris : V. Courdaveaux.

Gourevitch, A. J. (1983). Les Catégories de la culture médiévale. Paris : Gallimard.

Halász, K. (1992). Images d’auteur dans le roman médiéval, XII et XIII siècles. Debrecen : Kossuth Lajos Tudomanyegyetem.

Már Jónsson, E. (2006). Les 'Miroirs aux princes’ sont-ils un genre littéraire ? Médiévales, 51, 153-166.

Meschonnic, H. (1976). Les Proverbes, actes de discours. Revue des Sciences Humaines, XLI, $163,419-430$.

Minet-Mahy, V. (2007). Le Songe. De la mort de l'auteur à la naissance du lecteur/auditeur. Dans Corbellari, A., Tilliette, J.-Y. (éd.). Le Rêve médiéval (p. 193-220). Genève : Droz.

Morawski, J. (1925). Proverbes français antérieurs au XVe siècle. Paris : Champion.

Ollier, M.-L. (2000). La Forme du sens, Textes narratifs des XII et XIII e siècles, études littéraires et linguistiques. Orléans : Paradigme.

Parrot, F. (1995). L'Homme qui rêve. De l'anthropologie du rêve à la neurophysiologie du sommeil paradoxal. Paris : PUF.

Pastoureau, M. (1984). Quel est le roi des animaux ?. Dans Le Monde animal et ses représentations au Moyen-Âge (XI $-X V^{e}$ siècles). Actes de la Société des Historiens médiévistes de l'Enseignement Supérieur public, $15^{\mathrm{e}}$ congrès (p. 133-142). Toulouse. Récupéré de www.persee.fr/doc/shmes_1261-9078_1985_act_15_1_1442

Pastoureau, M. (2004). Une Histoire symbolique du Moyen Âge occidental. Paris : Seuil.

Pastoureau, M. (2007). L'Ours, histoire d'un roi déchu. Paris : Seuil.

Pastoureau, M. (2011). Bestiaires du Moyen Âge. Paris : Seuil.

Schulze-Busacker, É. (1985). Proverbes et expressions proverbiales dans la littérature narrative du Moyen Âge français : recueil et analyse. Paris : Champion.

Schulze-Busacker, É. (1997). La Place du proverbe dans la mentalité médiévale. Paremia, 6, 565-576.

Van Dijk, T. A., Kintsch, W. (1983). Strategies of discourse comprehension. New York: Academic press.

Van Gorp, H. et al. (2005). Dictionnaire des termes littéraires. Paris : Champion. 
RÉSUMÉ : Florimont, d'Aimon de Varennes, un trouvère lyonnais du XII siècle, semble avoir été écrit en 1188, mais sa datation demeure incertaine. Ce roman narre comment un jeune homme, profondément épris d'une fée, est amené à trahir malgré lui celle qu'il aime, perdant ainsi son amour et comment, après bien des souffrances, le jeune héros se console auprès d'une autre et devient roi. Tout au long des aventures de Florimont, son maitre Flocart l'accompagne, le guide et l'éduque par des discours fortement moralisateurs. Nous nous proposons d'étudier comment ce personnage, qui, selon Katalin Halász, participe d'un pouvoir supérieur ou de l'omniscience du narrateur, construit sa relation avec son élève, dans un but d'édification individuelle et collective. Nous verrons tout d'abord que Flocart assume une double fonction diégétique qui favorise le processus d'individuation de son élève. Nous montrerons ensuite que ses multiples interprétations des rêves lui confèrent le rôle de maitre de vérité qui sacralise le destin de Florimont. Nous observerons, enfin, que les discours parémiologiques de Flocart prennent des allures de miroir aux princes propre à révéler à son jeune élève les principales qualités d'un bon roi en plaçant la largesse au cour des relations humaines et politiques.

Mots-clés : Flocart, Florimont, Aimon de Varennes, maître, élève, discours parémiologique, miroir au prince

\section{Flocart and Florimont, the mirror and the prince}

ABSTRACT: Florimont, by Aimon de Varennes, a 12th-century Lyonnais author, seems to have been written in 1188 , but its dating remains uncertain. The novel tells how a young man, deeply enamored of a fairy, is led to betray, in spite of himself, the one he loves, thus losing his love and how, after many sufferings, the young hero consoles himself with another love and becomes king. Throughout his adventures, Florimont is accompanied by his master, Flocart, who guides and educates him with highly moralistic speeches. We propose to study how the latter character, who, according to Katalin Halász, participates in a higher power or omniscience of the narrator, builds his relationship with his pupil, for the purpose of individual and collective edification. We will first see that Flocart assumes a dual diegetic function that favors the process of individuation of his pupil. We will then show that his multiple interpretations of dreams give him the role of a master of truth who sanctifies the destiny of Florimont. Finally, we will observe that Flocart's paremiological discourse takes on the appearance of a mirror for princes, able to reveal to his young pupil the principal qualities of a good king, which is achieved by placing generosity at the heart of human and political relations.

Keywords: Flocart, Florimont, Aimon de Varennes, master and student, paremiological speech, mirror for princes 